\title{
Immunoextraction of pesticides at the trace level in environmental matrices
}

\author{
M. Bouzige and V. Pichon
}

École Supérieure de Physique et de Chimie Industrielles (ESPCI), Laboratoire Environnement et Chimie Analytique (ERS CNRS 657), 10 rue Vauquelin, 75231 Paris Cedex 05, France

\begin{abstract}
The trace-level determination of a large variety of compounds requires high performance from analytical separation and detection as well as from sample preparation technique. The coupling of a preconcentration step using solid-phase extraction and liquid chromatography is now widely used in environmental analysis. However one factor limitating the sensitivity of the method is due to the lack of selective sorbents. Low detection limits cannot be obtained in complex environmental matrices owing to many interfering compounds co-extracted and co-eluted with classical reversed-phase sorbents, i.e. octadecylsilicas and apolar copolymers.

Immunoaffinity sorbents (ISs) have been synthetized for the extraction of several related compounds within different classes of pesticides, such as phenylureas and triazines. These sorbents have been obtained by covalent bonding of polyclonal antibodies to a silica matrix. The antibodies present a strong affinity for the antigen and a more or less strong one for other structurally-related analytes including their degradation products. The high degree of selectivity of these sorbents allows to reach the detection limits according to the regulatory guidelines in the various studied matrices.
\end{abstract}

analyte and its metabolites which are structurally related to the parent molecule.

In contrast to immunoassays, ISs provide individual quantification of each analytes, because, once the compounds have been recognized and extracted onto the IS, they are desorbed, separated and analyzed. Therefore the cross-reactivity which can be a problem for immunoassays can be used in this case for the selective extraction of a whole group of structurally related compounds.

The first commercial ISs were introduced for the cleanup of samples for the determination of aflatoxins. Other ISs have been described in the literature for the analysis of single pesticides such as carbendazim, chlortoluron, atrazine or terbutylazine [1-4]. However, because of the unavoidable cross-reactivity of antibodies tailored for small molecules, ISs were also developed for the selective extraction of a whole group of structurally related compounds such as the triazine and phenylurea pesticides including their metabolites [5-11]. The class-selective trapping is now recognized as a key feature of ISs to be tailored for environmental analysis of pesticides. There is also an interest to develop IS for single analytes which are particularly difficult to analyze at trace-level because of the lack of available extraction methods from water such as aminotriazole.

\section{Synthesis of the immunosorbents}

The overall process for the preparation of an immunosorbent first involves the production of antibodies. Low molecular mass compounds such as pesticides are unable to evoke an immune response. Then production of antibodies towards small molecules (haptens) involves the attachment of this molecule (or an analog of it) to a larger molecule, as a protein (bovin serum albumin, $\beta$-lactoglobuline, ...), to form an immunogen that can be used for the immunization. The design of the hapten is the most crucial step in the development of an immunochemical technique for small molecules. Many reviews have shown that an appropriate hapten design determines the features of the resulting antibodies [12-16]. The optimum hapten for a selected target analyte has to be a near-perfect mimic of this molecule, in structure, geometry, electronic and H-bonding capabilities and hydrophobic properties [17]. Moreover, the strategy will be different whether a single compound within a class is targeted or the whole class. For the class-selective assay, the handle will be best located at a position which leaves the common sites exposed to the immune system.

New technologies for developing specific antibodies are an active area, using sometimes very sophisticated methods, 
but one has to realize that up to date, commercial Abs use mainly polyclonal or monoclonal antibodies. In both cases, the different steps, required to produce Abs, are similar up to the immunization.

Polyclonal antibodies (pAbs) are obtained by immunizing the host animal with the immunogen. Then, the obtained serum contains a mixture of antibodies with various affinities called polyclonal Abs and this serum has to be purified in order to obtain the IgG fraction containing $15 \%$ of active antibodies specific of the target molecule.

If pAbs have proven to be performant and are used in many commercial kits, they have some limitations because polyclonal antiserum can vary from one animal to another and the supply of Abs ends when the animal dies. This can be overcome by the use of the hybridoma technique based on the fusion of spleen lymphocytes (that produce Abs) isolated from immunized mice with myeloma cells [18]. The advantage in this technology is an unlimited production of monoclonal Abs (mAbs) with constant affinities. However, in contrast to pAbs production, this technique is difficult, laborious, time-consuming and expensive. Nevertheless, $\mathrm{mAbs}$ are now introduced in commercial immunoassays for their long-term supply. Because they have constant characteristics so that the performances of the kits are expected to be very reproducible.

After the antibody purification to obtain the IgG fraction, the ISs are produced by binding antibodies onto a solid support. Immobilization of antibodies can be done by different ways, such as physical or affinity adsorption but the covalent binding appears to be the best process of immobilization to obtain a good stability between the sorbent and the antibodies.

The selected sorbent should (i) have large size pores because antibodies are large molecules, (ii) be hydrophilic in order to avoid any non-specific interactions and (iii) be pressure resistant to be used in on-line techniques where the IS is directly coupled with LC [7-8]. The third requirement is not obligatory for off-line disposable cartridges and agarose is often used for antibody immobilization [19]. Only silica-based sorbents meet the three features, and in order to reduce the preparation steps, it is more rapid to use commercial silicas already modified by appropriate functional groups.

Many authors have attempted to use hydrophilic polymers because they allow an oriented bonding of the antibodies and they have higher capacities. However, hydrophilic polymers all contain $\pi$-bonds which obligatory give rise to non-specific hydrophobic interactions and decrease dramatically the selectivity of the ISs, which is their primary interest in sample preparation methods.

\section{Description of an immunosample pretreatment procedure}

If the IS is packed in a disposable cartridge, the sequence is very similar to that used on conventional $\mathrm{C}_{18}$ silica cartridge. A typical sequence is described in figure 1 .

After a conditioning step using PBS (phosphate buffer saline solution), the sample is percolated through the IS. A

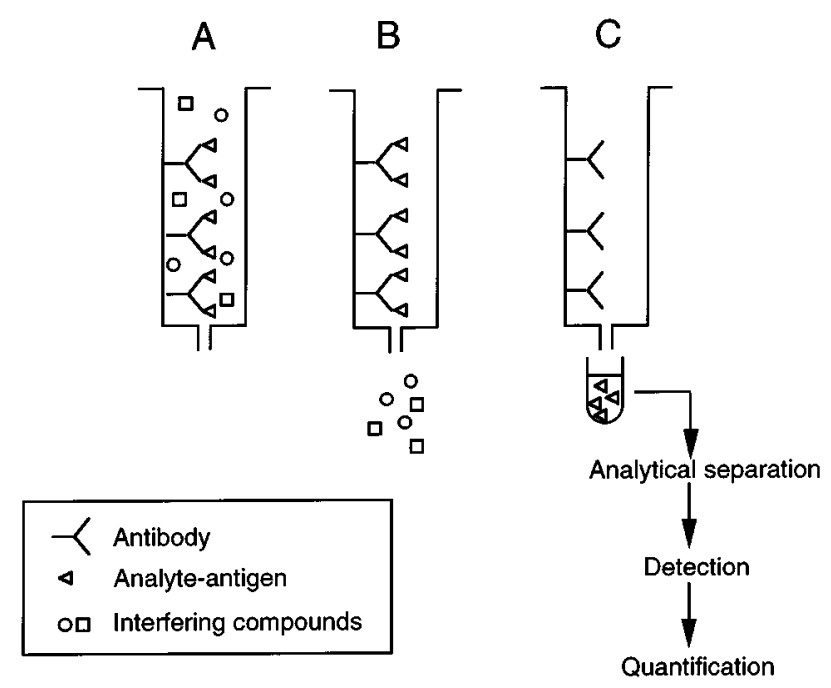

Figure 1. Immunosample pretreatment procedure.

washing step using a few $\mathrm{mL}$ of pure water or a small amount of organic solvent can be used to eliminate compounds which did not react with antibodies. The desorption of the compounds retained by the antibodies is achieved by the percolation of a few $\mathrm{ml}$ of eluant. The obtained residue can be reduced and analyzed by liquid chromatography (LC) or gas chromatography.

Desorption from ISs mainly occurs by flushing a saline buffer or a chaotropic solution. However, it has been shown that low molecular weight compounds were not desorbed using totally aqueous solutions or only when using a large volume of this solution (that have to be further reconcentrated). The desorption of small molecules from the IS required the addition of a certain amount of organic solvents in water. Desorption occurs because immobilized Abs are denatured upon flushing this mixture, but it has been shown that this denaturation is reversible and IS can be easily regenerated with subsequent washing with water or buffer [19]. Desorption conditions appear to depend on the strength of the binding interaction between compounds of interest but generally $3-4 \mathrm{~mL}$ of $70-80 \%$ of methanol or acetonitrile in water can be used.

When using a covalent immobilization of Abs onto silica, the obtained IS is stable and pressure resistant and can directly be used in an on-line system. In this case, the IS is packed in a precolumn which is placed in the sample-loop position of a six-port switching valve. After the percolation of the aqueous sample through the precolumn, by switching the valve in the inject position, the $C_{18}$ analytical column is directly coupled to the IS precolumn. The desorption of the retained compounds from the IS to the analytical column is ensured by the mobile phase that contains a sufficient amount of organic solvent. Results obtained with this on-line system using a silica-based material have shown a good efficiency for the coupling of this IS to the LC system. Such a system can be easily automated and therefore increases the potential of this method in terms of time and reproducibility. This allows also to limit the risk of loss and of contamination of the sample. 


\section{Potential for class-specific immunosorbents}

In order to make IS capable of trapping the highest number of compounds within a group, it is important to have a close look at the structure of these compounds. There again, the hapten design is important. Table I shows the potential for class-specific ISs obtained for phenylureas when bonding polyclonal anti-chlortoluron antibodies and for triazines when bonding anti-atrazine or anti-simazine antibodies by reporting recoveries of extraction. The measurement of extraction recoveries obtained for a given volume of sample (calculated by the ratio between the retained amount on the IS comparing to the percolated amount) is a good mean to define the cross-reactivity of antibodies and then to characterize the antigen-antibody interaction.

For the group of phenylureas, good recoveries were obtained on the anti-chlortoluron IS for almost all the compounds except fenuron which has no substituent on the phenyl ring and fluometuron. For the other compounds, most of them are characterized by one or two substituents (as chlortoluron) and then are generally well recognized by the Abs and then well retained by this IS. For triazines, it was predicted that the anti-atrazine antibody should be unable to cross-react with de-isopropylatrazine and that was experimentally verified, whereas the anti-simazine cross-reacts with this analyte. Therefore, if one wants to trap all the triazine class including metabolites, it is appropriate to mix the two ISs obtained with anti-atrazine and anti-simazine antibodies in a single cartridge or precolumn.

Recoveries reported in table I have been measured with a mixture of compounds, each one being present at the same concentration in the percolated sample. We can expect the lowest recoveries to be obtained for compounds having the lowest affinity for the antibodies. In a solid-phase extraction process, an incomplete recovery is explained by analyte breakthrough which can be due to an insufficient retention (i.e. a low affinity of the Abs for the compound) or to the overloading of the capacity of the IS. With $\mathrm{C}_{18}$ silicas, the capacity is high and when compounds are present at the $\mu \mathrm{g} / \mathrm{L}$ level, breakthrough mainly occurs because of insuffi- cient retention. It was shown that the recovery of a given compound can be predicted from the breakthrough volume of the analytes and from the percolated sample volume. As for classical SPE sorbent, it is then important to define the percolated volume from which depend the recoveries of extraction of all the pesticides.

But low recoveries can also be explained by an overloading of the capacity. For quantitative purpose, it is important to know how works an IS and the relation that exists between recovery, capacity and affinity order. In other words, since there is a competition reaction among the various analytes in the group, the question is whether recoveries depend on the number of related analytes in the sample and on their respective concentration.

This parameter is usually defined as the total number of immobilized active antibodies then as the part of specific IgGs immobilized on the IS and that can trap compounds of interest. In many studies, IS capacity is determined using the pesticide-antigen since the antibody affinity is expected to be the highest for this compound. However, owing to the affinity chromatography process, this capacity cannot be directly transposed to the other compounds within the group. The capacity is usually lower for related analytes than for the pesticide-antigen. Studies have shown that, to be sure that the capacity is enough for the simultaneous extraction of several compounds in the same sample, one has just to verify that the calibration curves are linear for the whole range of concentration and for all the studied compounds. That means that, for a given volume, recoveries of extraction for all the pesticides are constant and do not depend on the number of pesticides that can be present together in the sample.

\section{Selectivity and limits of detection}

\section{In water}

The limit of detection that can be reached for a given compound using ISs depends on the recovery of extraction

Table I. Potential for class-specific immunosorbents measured by recoveries of extraction obtained for phenylureas and triazines. Recoveries measured by percolation of $50 \mathrm{~mL}$ of water spiked wih $0.5 \mu \mathrm{g} / \mathrm{L}$ of each phenylurea and of $25 \mathrm{~mL}$ of water spiked with $3 \mu \mathrm{g} / \mathrm{L}$ of each triazine. Adapted from reference [8].

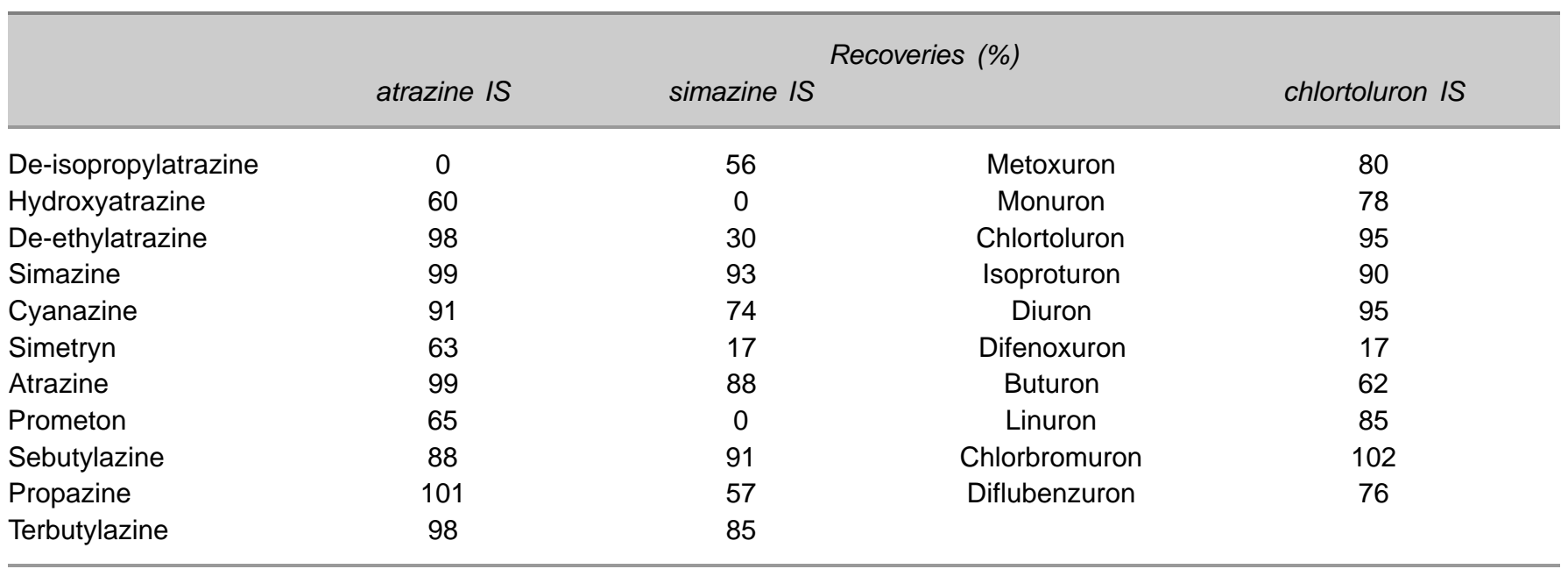


obtained and consequently on the affinity developed by the immobilized antibodies towards this compound. However, the selectivity of the interaction is so high that sample volumes can be reduced (compared to volumes preconcentrated on non selective sorbents) thus allowing to obtain good recoveries for most of the compounds. Figure 2 shows the chromatograms corresponding to the on-line preconcentration on an anti-chlortoluron IS of $50 \mathrm{~mL}$ of LC-grade water spiked at $0.1 \mu \mathrm{g} / \mathrm{L}$ (Fig. 2a) and river Seine water spiked at $0.5 \mu \mathrm{g} / \mathrm{L}$ (Fig. 2b) with a mixture of phenylureas and the chromatograms corresponding to the off-line preconcentration on an anti-atrazine IS of $50 \mathrm{~mL}$ of river Seine water non spiked (Fig. 2c) and spiked at $0.1 \mu \mathrm{g} / \mathrm{L}$ (Fig. 2d) with a mixture of triazines. The high selectivity of the immunoaffinity system allows the easy detection of these pesticides at low level of concentration, even in the highly contaminated river water sample.

It is worthwhile to note that the extraction procedure is similar for the two classes (i.e. conditioning with a buffer, sample application, and desorption with a few $\mathrm{mL}$ of methanol-water mixture, 70:30, v/v or on-line desorption with the acetonitrile-water gradient) so that multi-residue extraction can be obtained for these two classes using a single extraction cartridge packed with a mixed bed of anti-triazine and anti-phenylurea ISs. This was done by mixing an anti-atrazine IS and an anti-chlortoluron IS and applied to the simultaneous extraction of the two classes of pesticides from river Seine water spiked at $0.5 \mu \mathrm{g} / \mathrm{L}$ with each analytes (Fig. 3b). The comparison of this chromatogram with the chromatogram corresponding to the on-line preconcentration of the same sample on a non selective apolar copolymer of PLRP-S (Fig. 3a) shows also the high selectivity of this mixed-bed IS that allows the easy detection of all the pesticides including the polar metabolite of atrazine, i.e. de-ethylatrazine, which is co-eluted with interfering polar compounds extracted on PLRP-S.

\section{In food}

With regards to solid matrices such as food, the immunosorbents have been shown to be very simple and very effective in cleaning up extracts for trace concentrations of pesticides before determination by standard LC or GC procedure. In addition, it offers the potential of reducing the use of organic solvents normally used for purification. Currently, the extracts are obtained after classical solvent extraction methods and, after the evaporation of the extraction solvent, the extracts are simply diluted in water to be preconcentrated and cleaned up on the ISs.

Recently, ISs were prepared and evaluated for the determination of phenylureas [9] and triazines [10] in several food samples (carrot, celery, corn, grapes, onions, potatoes,
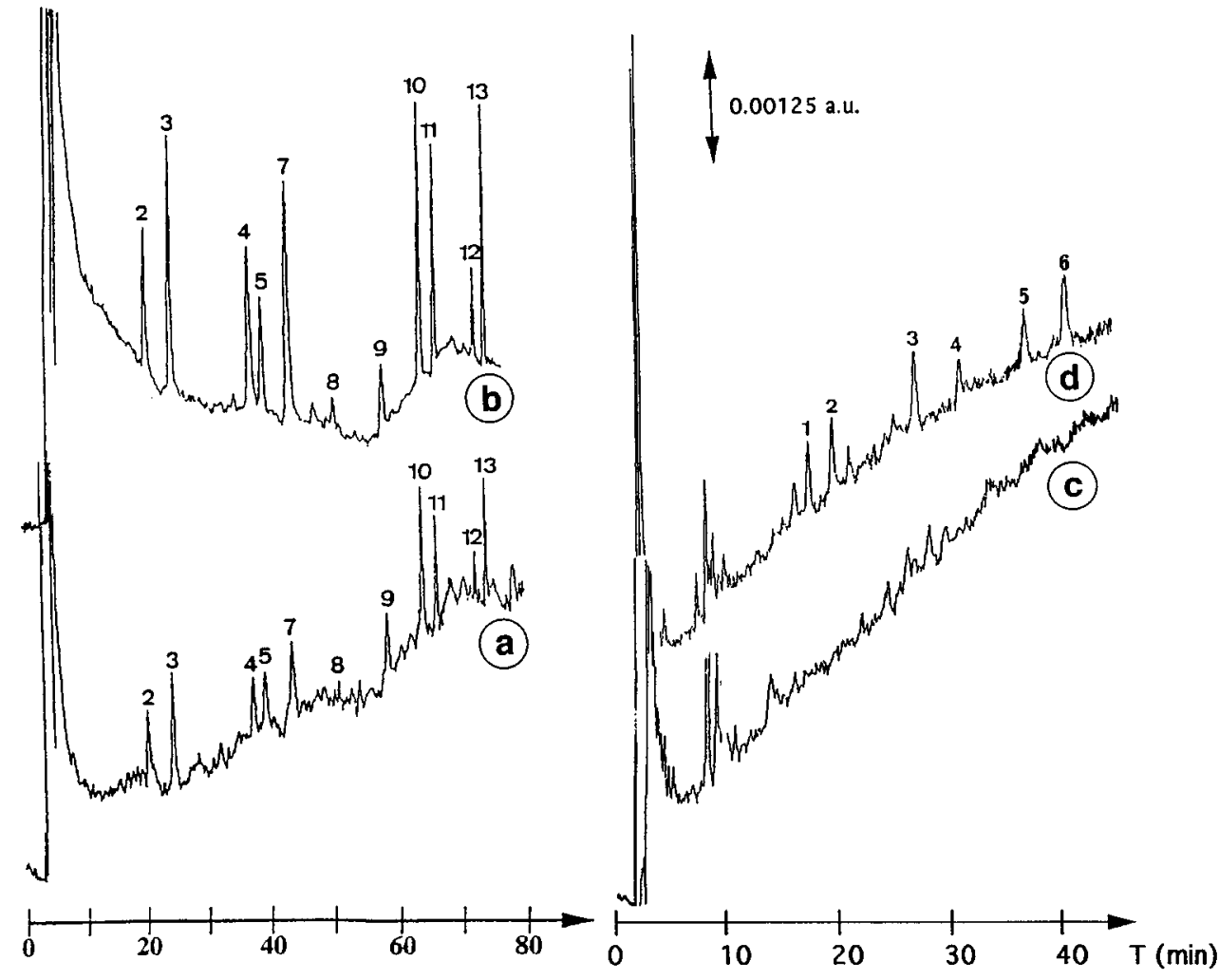

Figure 2. On-line preconcentration of $50 \mathrm{~mL}$ of LC-grade water a) and river Seine water b) spiked at 0.1 and $0.5 \mu \mathrm{g} / \mathrm{L}$ with a mixture of phenylureas respectively on an anti-chlortoluron IS and off-line preconcentration of $50 \mathrm{~mL}$ of river Seine water non-spiked c) and spiked at $0.1 \mu \mathrm{g} / \mathrm{L}$ d) with a mixture of triazines. Reversed-phase LC separation with water-acetonitrile gradient. UV detection at $244 \mathrm{~nm}$ for phenylureas and $220 \mathrm{~nm}$ for triazines. Phenylureas: (1) Fenuron, (2) Methoxuron, (3) Monuron, (4) Methabenzthiazuron, (5) Chlortoluron, (6) Fluometuron, (7) Isoproturon, (8) Difenoxuron, (9) Buturon, (10) Linuron, (11) Chlorbromuron, (12) Diflubenzuron, (13) Neburon. Triazines: (1) Simazine, (2) Cyanazine, (3) Atrazine, (4) Prometon, (5) Propazine, (6) Terbuthylazine. 
apples, peas and strawberries). The detection limits were estimated to be $2-10 \mathrm{ng} / \mathrm{g}$ depending upon the compounds and the plant tissue analyzed. To illustrate the high selectivity of this immunoclean-up, a chromatogram corresponding to the direct injection of a standard mixture of triazines (Fig. 4a) is compared to the chromatogram corresponding to an extract of carrot spiked at $25 \mathrm{ng} / \mathrm{g}$ and passed through an anti-atrazine IS (Fig. 4b). The baselines of the two chromatograms are very similar allowing an easy detection and quantification of these triazines in this extract of carrots.

\section{In soil or sediment}

This selectivity can also be applied to simplify the analysis of soil extracts. This was done for phenylureas or triazines and their metabolites in soil [11,20]. The chromatogram, in figure $5 \mathrm{~b}$, corresponding to the analysis of the soil extract showed no interfering peaks and was very similar to the chromatogram corresponding to the analysis of a LC gradewater sample (Fig. 5a). The high selectivity of the pretreatment based on the use of an anti-chlortoluron IS allows the easy quantification of these phenylureas. This is also illustrated by the identification and quantification of linuron in the non spiked soil (Fig. 5c) that was naturally contaminated by $66 \pm 3 \mathrm{ng} / \mathrm{g}$ of this compound. Without this clean-up step, the direct analysis of this type of matrix extract is characterized by many interfering peaks and a huge hump in the chromatogram that does not allow the easy quantification of compounds and particularly of polar metabolites [11,20].

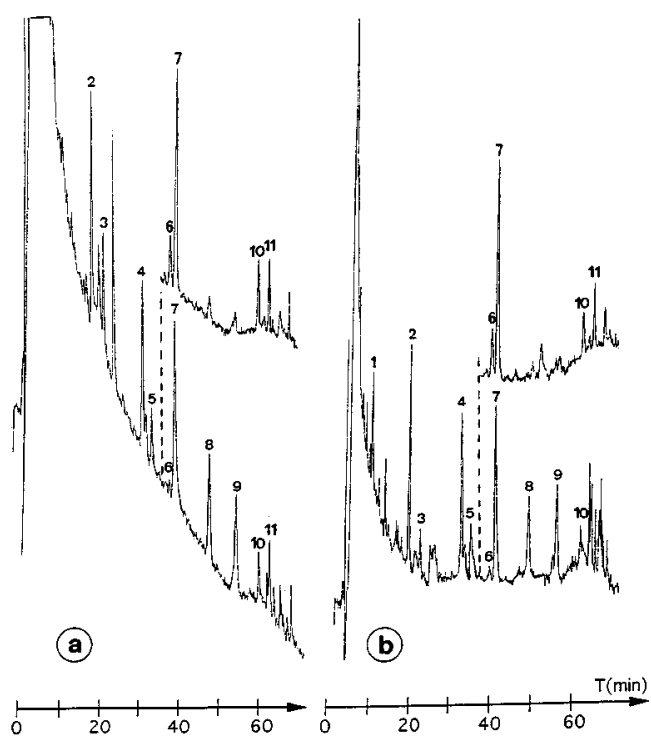

Figure 3. Multiresidue extraction of triazines and phenylureas from $50 \mathrm{~mL}$ of river Seine water (spiked at $0.5 \mu \mathrm{g} / \mathrm{L}$ with each compound) on a non-selective PLRP-S sorbent a) and on a mixed-bed IS based on anti-atrazine and antichlortoluron antibodies b). Reversed-phase LC separation with water-acetonitrile gradient. Detection at $220 \mathrm{~nm}$ and partly at $244 \mathrm{~nm}$. Compounds: (1) De-ethylatrazine, (2) Simazine, (3) Monuron, (4) Atrazine, (5) Chlortoluron, (6) Isoproturon (7) Diuron, (8) Propazine, (9) Terbutylazine, (10) Linuron, (11) Chlorbromuron.

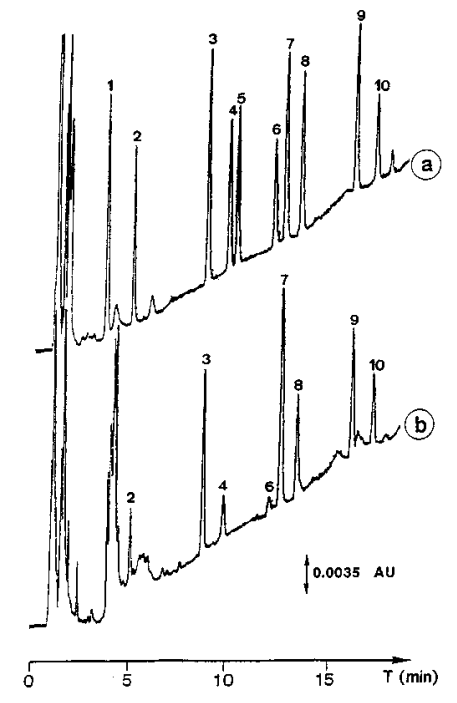

Figure 4 : Direct injection of a standard mixture of triazines (2.5 - $16 \mathrm{ng}$ of each triazine) a) and of a carrot extract (spiked to contain $56-100 \mathrm{ng} / \mathrm{g}$ of each triazine) passed through an antiatrazine IS b). Adapted from reference [10]. Reversed-phase LC separation with water-methanol gradient. Detection at $220 \mathrm{~nm}$. Compounds: 1, Hydroxy-atrazine; 2, De-ethylatrazine; 3, Simazine; 4, Cyanazine; 5, Metribuzin; 6, Simetryn; 7, Atrazine; 8, Prometon; 9, Propazine; 10, Terbutylazine.

\section{Validation studies}

A validation study was reported using an anti-isoproturon IS for the determination of phenylureas in water through an interlaboratory study using Aquacheck certified samples [21]. The overall relative standard deviation comparing the values obtained by this method and the real values given by Aquacheck organization varied between 1 and 22\%. All the samples were analyzed simultaneously by LC-DAD and LCAPCI-MS in order to confirm the identity of the compounds.

Another important feature of the ISs is their high stability. ISs can be easily regenerated using a phosphate buffer saline (PBS) solution. It was verified that after 50 runs, the loss in capacity was less than 10\% [7-8]. They can be submitted to high proportions of organic solvents, which destroy the antigen-antibody interactions, certainly by modification of the conformation of the protein, but in a reversible way, because antibodies have been stabilized by the covalent binding to the silica.

\section{Conclusion}

Class-specific immunosorbents are expected to be developed and become available in the near future for immunochemical sample preparation, as a consequence of the wider acceptance of immunoassays by analytical chemists. Other immunosorbents are now in development for different groups of new pollutants in research laboratories. Commercialization of these immunosorbents will require the availability of reproducible antibodies, which can be better 


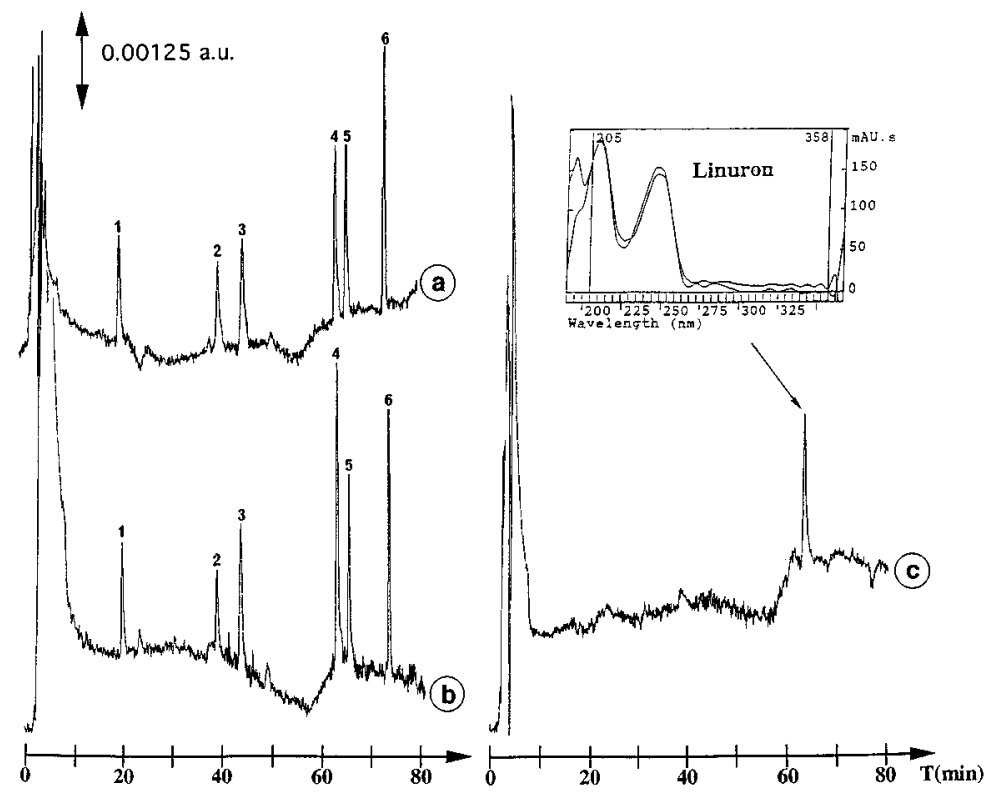

Figure 5. On-line preconcentration on an anti-chlortoluron IS of $25 \mathrm{~mL}$ of LC-grade water spiked with $25 \mathrm{ng}$ of each phenylurea a) and of an aqueous residue obtained after the solvent extraction of $0.33 \mathrm{~g}$ of soil spiked with $25 \mathrm{ng}$ of each phenylurea b) and non spiked containing linuron identified by its UV spectrum (c). Solutes: (1) Metoxuron, (2) Fluometuron, (3) Isoproturon, (4) Linuron, (5) Chlorbromuron, (6) Neburon. Reversed-phase LC separation with water-acetonitrile gradient. Detection at $244 \mathrm{~nm}$. Adapted from reference [11].

guarantied by monoclonal antibodies. Moreover, recent works have shown that the cross-reactivities of mAbs are similar to those of pAbs meaning that one can consider the possibility of making a selective extraction of pesticides by class using these antibodies.

\section{References}

1. Marx, A.; Giersch, T.; Hock, B. Anal. Lett. 1995, 28, 267.

2. Rule, G. S.; Mordehal, A. V.; Henion, J. Anal. Chem. 1994, 66, 230-235.

3. Thomas, D. H.; Beck-Westermeyer, M.; Hag D.S., Anal. Chem. 1994, 66, 3823-3829.

4. Shahtaheri, S. J.; Katmeh, M. F.; Kwasowski, P.; Stevenson, D. J. Chromatogr. A 1995, 697, 131-136.

5. Hage, D. S.; Rollag, J. G.; Thomas, D. H. Preprint of papers presented at the 211th ACS National meeting, New-Orleans, March 24-28, 1996; p 101.

6. Pichon, V.; Chen, L.; Daniel, R.; Martel, A.; Le Goffic, F.; Abian, J.; Barcelo, D.; Hennion, M. C. Anal. Chem. 1995, 67, 2451-2460.

7. Pichon, V.; Chen, L.; Hennion, M. C. Anal. Chim. Acta 1995, 311, 429-436.

8. Pichon, V.; Chen, L.; Durand, N.; Le Goffic, F.; Hennion, M. C. J. Chromatogr. A 1996, 725, 107-119.

9. Lawrence, J. F.; Menard, C.; Hennion, M. C.; Pichon, V.; Le Goffic, F.; Durand, N. 1996, 732, 277-281.

10. Lawrence, J. F.; Menard, C.; Hennion, M. C.; Pichon, V.; Le Goffic, F.; Durand, N. J. Chromatogr. A 1996, 752, 147-154.
11. Pichon, V.; Rogniaux, H.; Fischer-Durand, N.; Ben Rejeb, S.; Le Goffic, F.; Hennion, M. C. Chromatographia 1997, 45, 289-295.

12. Schlaeppi, J. M.; Föry, W.; Ramsteiner, K. J. Agric. Food Chem. 1989, 37, 1532-1538.

13. Goodrow, M. H.; Harrison, R. O.; Hammock, B. D. J. Agric. Food Chem. 1990, 38, 990-996.

14. Harrison, R. O.; Goodrow, M. H.; Hammock, B. D. J. Agric. Food Chem. 1991, 39, 122-128.

15. Muldoon, M. T.; Huang, R. N.; Hapemen, C. J.; Freis, G. F.; Ma, M. C.; Nelson, J. O. J. Agric. Food Chem. 1994, 42, 747755.

16. Eremin, S. A.; Samsonova, Zh.V.; Egorov, A. M. Russian Chem. Rev. 1994, 63(7) 611-622.

17. Goodrow, M. H.; Sandborn, J. R.; Stoutamire, D. W.; Gee, S. J.; Hammock, B. D.; Immunoanalysis of Agrochemicals, Emerging Technologies, ACS Symp. Ser., Vol. 586, American Chemical Society, Washington, 1995; pp 119-139.

18. Hock, B.; Giersh, T.; Kramer, K. Analysis 1992, 20(8), M29M33.

19. Farjam,A. in: The use of immobilized antibodies for the selective on-line sample pre-treatment in liquid- and gas chromatography, PhD thesis, Free University of Amsterdam, Amsterdam, The Nederlands, April, 1991.

20. Pichon, V.; Aulard-Macler, E.; Oubihi, H.; Sassiat, P.; Hennion, M. C.; Caude, M.; Chromatogr. 1997, 49, 529-536.

21. Ferrer, I.; Pichon, V.; Hennion, M. C.; Barcelo, D. J. Chromatogr. A 1997, 777, 91. 